\title{
GMR
}

\section{Bioinformatic and expression analysis of the OMT gene family in Pyrus bretschneideri cv. Dangshan Su}

\author{
X. Cheng, Y. Xiong, D.H. Li, J. Cheng, Y.P. Cao, C.C. Yan, Q. Jin, N. Sun, \\ Y.P. Cai and Y. Lin \\ School of Life Science, Anhui Agricultural University, Hefei, Anhui, China \\ Corresponding author: Y.P. Cai \\ E-mail: ypcaiah@163.com \\ Genet. Mol. Res. 15 (3): gmr.15038664 \\ Received March 29, 2016 \\ Accepted May 18, 2016 \\ Published September 2, 2016 \\ DOI http://dx.doi.org/10.4238/gmr.15038664
}

Copyright (C) 2016 The Authors. This is an open-access article distributed under the terms of the Creative Commons Attribution ShareAlike (CC BY-SA) 4.0 License

\begin{abstract}
With high nutritional value in its fruits, Dangshan $\mathrm{Su}$ pear has been widely cultivated in China. The stone cell content in fruits is a key factor affecting fruit quality in pear, and the formation of stone cells has been associated with lignin biosynthesis. O-Methyltransferase (OMT) is a key enzyme involved in lignin metabolism within the phenylpropanoid pathway. Here, we screened $26 O M T$ genes from the Pyrus bretschneideri cv. Dangshan Su genome using the DNATOOLs software. To characterize the $O M T$ gene family in pear, gene structure, chromosomal localization, and conserved motifs of PbOMTs were analyzed. $P b O M T$ s were divided into two categories, type I (designated PbCCOMTs) and type II (designated PbCOMTs), indicating the differentiation of function during evolution. Based on the analysis of multiple sequence alignment, cis-element prediction, and phylogenetic relationships, two candidate genes, PbCCOMT1 and PbCCOMT3, were selected for the analysis of temporal and spatial gene expression
\end{abstract}

Genetics and Molecular Research 15 (3): gmr.15038664 
in pear. The promoter regions of both $P b C C O M T 1$ and $P b C C O M T 3$ contain regulatory motifs for lignin synthesis. Moreover, the two genes show high similarity and close phylogenetic relationships with CCOMTs in other species. Expression analysis showed that transcript levels of two $P b C C O M T$ s were positively associated with the contents of both stone cells and lignin during the development of pear fruit. These results suggest that PbCCOMT1 and PbCCOMT3 are closely associated with lignin biosynthesis. These findings will help clarify the function of $P b O M T \mathrm{~s}$ in lignin metabolism and to elucidate the mechanisms underlying stone cell formation in pear.

Key words: $O M T$ gene family; Pyrus; Lignin synthesis; PbCCOMTs; Phylogenetic analysis

\section{INTRODUCTION}

Pyrus bretschneideri cv. Dangshan Su originated in Dangshan County, Anhui Province and is widely planted in China. As its fruit is very nutritious and has important medicinal value, this variety is in great demand both at home and abroad. In recent years, the number of stone cells in the fruits of Dangshan Su pears has increased as a result of the deterioration of field environments and field management (Cai et al., 2010; Jin et al., 2013). Stone cells are formed by parenchyma cells following the secondary thickening and lignification of primary walls. This acts as a type of sclerenchymatous cells in the pear pulp, which determines the internal quality of pear fruits (Cai et al., 2010; Jin et al., 2013). An increased concentration of stone cells in pear fruit leads to a muricated taste, poor edible quality, and loss of economic value (Choi and Lee, 2013; Zhao et al., 2013; Yan et al., 2014). During the formation of stone cells, lignin is deposited in the corner of the primary wall of parenchyma cells, from where it extends to the intercellular and secondary wall layers along the primary wall corner until the whole cell lumen is covered. Ultimately, a solid stone cell is formed (Lu et al., 2011; Jin et al., 2013). Therefore, lignin biosynthesis is thought to be involved in the generation of enlarged stone cells in pear fruit and in their presence in increased numbers. As a key enzyme in the phenylpropane pathway, O-methyltransferases (OMTs) are involved in the synthesis of a series of secondary metabolites such as lignins, flavonoids, and phytoalexins. OMTs can be divided into two types: type I, a caffeoyl-CoA-3-O-methyltransferase (CCOMT), which is a key enzyme involved in lignin biosynthesis; and type II, a caffeic acid O-methyltransferase (COMT), which is involved in lignin metabolism and flavonoid methylation (Joshi and Chiang, 1998). During lignin biosynthesis and using caffeoyl-CoA as a substrate, CCOMT transfers the methyl group from S-adenosylmethionine (SAM) to the lignin precursor benzene at the third carbon position, generating feruloyl-CoA. Conversely, COMT is responsible for methylation of lignin precursors such as coniferaldehyde and 5-hydroxyconiferaldehyde (Joshi and Chiang, 1998).

Both types of $O M T$ s exist in plants, and individual members of this family have different functions. In mutant screens, the Atomt1 mutant of Arabidopsis thaliana can be identified by its reduced levels of syringyl (S) units and flavonoid, and its increased levels of 5-hydroxyguaiacyl, which is a precursor of S-units. These results suggest that AtOMT1

Genetics and Molecular Research 15 (3): gmr.15038664 
is associated with the metabolism of both lignin and flavonoids (Goujon et al., 2003). An Arabidopsis mutant (ccomt1) was found to have decreased lignin content in the stem and an increased ratio of S/G lignin units. An Arabidopsis double mutant (comt1 ccomt1) showed lower levels of G units than were found in wild-type Arabidopsis (Do et al., 2007).

CCOMT genes have been targeted in order to lower the total lignin content while increasing the ratio of $\mathrm{S} / \mathrm{G}$ lignin units via RNA interference technology. For example, the suppression of $\operatorname{PrCCOMT}$ expression by interference RNA led to a $20 \%$ reduction in the total lignin content (Wagner et al., 2011). Suppression of CCOMT expression in maize showed that the lignin content was decreased and the ratio of S/G lignin units was increased (Li et al., 2013). Research showed that heterologously expressed anti-AcCCOMT increased the levels of $\mathrm{S}$ units, $\mathrm{G}$ units, and total lignin in tobacco (Pang et al., 2014). The results of previous studies have shown that $\mathrm{PbCCOMT}$ genes are expressed at high levels during the early stages of fruit development, which might promote the accumulation of pear stone cells (Wu et al., 2012). Furthermore, a higher ratio of $\mathrm{G}$ units can lead large trouble in degradation of polymeride lignin, which subsequently increases the degree of sclereid polymerization, and seriously affects the quality and taste of pear fruit (Yan et al., 2014). This indicates that CCOMT is a valuable regulatory gene.

van Parijs et al. (2015) analyzed the phylogenetic evolution of COMTs in Lolium perenne. Wang et al. (2013) screened nine OMT genes involved in lignin synthesis from the whole genome of Eucalyptus using methods such as RNA-seq, qRT-PCR, and a composite evolutionary tree. However, no recent studies on the $O M T$ gene family in pears have been performed using genome-wide methods. In the present study, based on the published pear genome sequence, the $O M T$ gene family in pear was investigated to analyze its chromosome distribution, phylogenetic relationships, conserved motifs, and the expression pattern of candidate $P b C C O M T s$ relevant to lignin biosynthesis at the genome-wide level. These results should provide some useful information for further studies on the functions of PbOMTs, developmental mechanisms of stone cells, and regulation of pear lignin metabolism.

\section{MATERIAL AND METHODS}

\section{Identification and sequence analysis of $O M T$ family genes in pear}

The pear genome sequence was downloaded from GigaDB (http://gigadb.org/ dataset/100083), and the DNATOOLs software was used to construct a local database from the protein sequences of the latest complete pear genome. Two Arabidopsis OMTs (At5g54160 and At4g34050), which are relevant to lignin biosynthesis, were used as initial query sequences to search the pear protein database using the BLASTp program $(\mathrm{P}=0.001)$. Redundant sequences were then removed manually. Hidden Markov Models of the Pfam (http://pfam.sanger.ac.uk/ search) and SMART (http://smart.embl-heidelberg.de/) databases were used to confirm each candidate sequence containing the signature methyltransf 2 (PF00891) or methyltransf 3 (PF01596) domains of $O M T$ genes. The molecular weight (MW) and isoelectric point (pI) of proteins were calculated with the Compute $\mathrm{pI} / \mathrm{Mw}$ tool (http://web.expasy.org/compute pi/) (Bjellqvist et al., 1993). Information on PbOMTs, including amino acid sequences, chromosome location, clone number, and open reading frame length was obtained from the pear genome sequence database.

Genetics and Molecular Research 15 (3): gmr.15038664 


\section{Phylogenetic analysis, conserved protein motifs, and prediction of gene structure}

Phylogenetic relationships were analyzed using MEGA 5.1 (Tamura et al., 2011) with the neighbor-joining $(\mathrm{NJ})$ method (bootstrap $=1000$ ). Information on proteins used for phylogenetic analysis is listed in Table S1.

The conserved motifs encoded by each $O M T$ gene were identified using the Multiple Em for Motif Elicitation (MEME) program (http://meme-suite.org/tools/meme) (Bailey et al., 2006). Parameters were set as follows: optimum motif width $\geq 6$ and $\leq 200$; maximum number of motifs 20. To predict the exon-intron structure of the PbOMT genes, the genomic sequences and their predicted coding sequences were compared using GSDS (http://gsds.cbi.pku.edu.cn/).

\section{Estimating $\mathrm{Ka} / \mathrm{Ks}$ ratios for duplicated gene pairs}

Duplicated $O M T$ gene pairs in pear were aligned by ClustalW. Next, the Ka (nonsynonymous substitution rate) and Ks (synonymous substitution rate) values were calculated using the DnaSP v5.0 software (Rozas and Rozas, 1995), and the Ka/Ks ratio was analyzed to assess the selection pressure for each gene pair.

\section{Chromosomal locations and gene duplications}

Individual $O M T$ genes were mapped on pear chromosomes according to their starting positions provided in the genome annotation document. The image showing chromosome location was generated by the MapInspect software (http://mapinspect. software.informer.com).

We defined gene duplication according to the following criteria: 1) the aligned sequence covered $>80 \%$ of the longer gene, 2 ) the identity of the aligned regions was $>80 \%$. Based on the chromosomal locations of the OMT genes, two types of gene duplications were recognized: tandem duplication and segmental duplication (Kong et al., 2013; Wei et al., 2013).

\section{Alignment of amino acid sequences encoded by $O M T s$ and analysis of their secondary components}

Protein sequences of pear and Arabidopsis were aligned using Iden and Sim in Sequence Manipulation Suite (Stothard, 2000). Multiple comparisons of each amino acid sequence were carried out using the BioEdit software and secondary elements were analyzed using the online software Espript3.0 (Robert and Gouet, 2014).

\section{Cis-element analysis and interaction networks}

For promoter analysis, 2000-bp genomic DNA sequences from upstream of the initiation codon (ATG) were retrieved from the pear genome sequence. Analysis of cisacting regulatory DNA elements (cis-element) was carried out using the PLANT CARE program (http://bioinformatics.psb.ugent.be/webtools/plantcare/html/) (Magali et al., 2002). Functional protein association networks were integrated in the STRING software (http://string-db.org/). 


\section{RNA extraction and reverse transcription}

To examine the expression of $P b O M T$ genes, buds, young stems, mature leaves, and fruits were obtained from 50-year-old pear trees grown on a farm in Dangshan, Anhui, China. All samples were stored in liquid nitrogen until use. Fruits were collected at different days after flowering (DAF) $(15,39,47,55,63$, and 145 DAF).

Total RNA was isolated from all samples using a total RNA isolation kit (Tiangen, China). Reverse transcription was performed using a M-MLV Rtase cDNA Synthesis Kit (TaKaRa, China).

\section{Primer design and semi-quantitative RT-PCR}

Primers were designed using Primer premier 5 and are listed in Table S2. Semiquantitative RT-PCR as performed as follows: predegeneration for $5 \mathrm{~min}$, followed by 30 cycles of degeneration for $30 \mathrm{~s}$ at $94^{\circ} \mathrm{C}$, annealing for $30 \mathrm{~s}$ at $55^{\circ} \mathrm{C}$, extension for $1 \mathrm{~min}$ at $72^{\circ} \mathrm{C}$, and a final $72^{\circ} \mathrm{C}$ extension for $10 \mathrm{~min}$. Each analysis was set up in triplicate.

\section{RESULTS}

\section{Identification of the $O M T$ genes in pear}

A total of 39 candidate genes were identified, and Pfam and SMART databases were subsequently used to confirm the presence of the methyltransf 2 or methyltransf 3 domain in all candidate genes. As a result, 26 members of the $O M T$ gene family were identified in the pear genome, including of 19 COMT genes and seven CCOMT genes, designated as PbCOMT1 to PbCOMT19, and PbCCOMT1 to PbCCOMT7, respectively (Table 1).

\begin{tabular}{|c|c|c|c|c|c|c|}
\hline Name & Sequence ID & Chromosome & Length (aa) & MW (kDa) & $\mathrm{pI}$ & ORF (bp) \\
\hline PbCOMT1 & Pbr013510.1 & Chr1 & 365 & 39.74 & 5.63 & 1096 \\
\hline PbCOMT2 & Pbr013512.1 & Chr1 & 377 & 41.00 & 5.67 & 1132 \\
\hline PbCOMT3 & Pbr040044.1 & Chr7 & 367 & 40.05 & 5.63 & 1102 \\
\hline PbCOMT4 & Pbr040039.1 & Chr7 & 365 & 39.59 & 5.75 & 1096 \\
\hline PbCOMT5 & Pbr040045.1 & Chr7 & 367 & 39.87 & 5.82 & 1102 \\
\hline PbCOMT6 & Pbr035407.1 & I & 358 & 39.48 & 5.28 & 1075 \\
\hline PbCOMT7 & Pbr025887.1 & Chr10 & 358 & 39.00 & 5.70 & 1075 \\
\hline PbCOMT8 & Pbr007791.1 & Chr1 & 370 & 41.03 & 5.78 & 1111 \\
\hline PbCOMT9 & Pbr025883.1 & Chr10 & 370 & 40.97 & 5.96 & 1111 \\
\hline PbCOMT10 & Pbr003001.1 & 1 & 291 & 32.35 & 6.16 & 874 \\
\hline PbCOMTII & Pbr021100.1 & Chr15 & 361 & 40.02 & 5.59 & 1084 \\
\hline PbCOMT12 & Pbr021099.1 & Chr15 & 363 & 40.19 & 6.08 & 1090 \\
\hline PbCOMT13 & Pbr021098.1 & Chr15 & 392 & 43.38 & 6.14 & 1177 \\
\hline PbCOMT14 & Pbr032564.1 & Chr12 & 369 & 40.53 & 5.26 & 1108 \\
\hline PbCOMT15 & Pbr030677.1 & Chr9 & 332 & 36.50 & 5.78 & 997 \\
\hline PbCOMT16 & Pbr040038.1 & Chr7 & 126 & 13.76 & 6.27 & 379 \\
\hline PbCOMT17 & Pbr030676.1 & Chr9 & 362 & 39.94 & 6.10 & 1087 \\
\hline PbCOMT18 & Pbr039628.1 & Chr10 & 236 & 26.51 & 6.25 & 709 \\
\hline PbCOMT19 & Pbr036056.1 & Chr17 & 358 & 39.39 & 5.38 & 1075 \\
\hline PbCCOMTI & Pbr034039.1 & Chr17 & 247 & 27.86 & 5.43 & 742 \\
\hline PbCCOMT2 & Pbr028157.1 & 1 & 149 & 16.34 & 7.66 & 448 \\
\hline РbCСOMT3 & Pbr038709.1 & 7 & 247 & 27.82 & 5.32 & 742 \\
\hline PbCCOMT4 & Pbr019305.1 & Chr9 & 235 & 26.70 & 5.43 & 706 \\
\hline PbCCOMT5 & Pbr028166.1 & 1 & 235 & 26.31 & 5.14 & 706 \\
\hline РbCCOMT6 & Pbr025246.1 & Chr5 & 307 & 34.12 & 8.91 & 922 \\
\hline PbCCOMT7 & Pbr015171.1 & Chr16 & 161 & 17.59 & 7.66 & 484 \\
\hline
\end{tabular}

"/" indicates that the specific chromosome is not known. MW: molecular weight; ORF: open reading frame. 
To characterize PbOMT proteins, their physicochemical properties (pI and MW) were analyzed. As shown in Table 1, most $P b C O M T$ sequences were longer and had a higher MW than those of PbCCOMT genes. PbCOMT proteins were between 126- and 392-aa long, with maximum and minimum molecular weights of 43.38 and $13.76 \mathrm{kDa}$, respectively, and isoelectric points between 5.26 and 6.27. PbCCOMT proteins are between 149- and 307-aa long, with maximum and minimum molecular weights of 34.12 and $16.34 \mathrm{kDa}$, and isoelectric points from 5.14 to 8.91 .

\section{Intron-exon structure and phylogenetic analysis of pear $O M T$ genes}

Based on the phylogenetic analysis (Figure 1), 26 OMT genes were divided into two major groups, with PbCOMTs in group I (seven genes) and PbCCOMTs in group II (19 genes). This implies that there were evolutionary differences between the two groups. Twentysix $O M T$ genes were clustered into eight gene pairs with high bootstrapping values, with the exception of $P b C C O M T 4 / 5$ and PbCOMT4/16. We further analyzed the structures of 26 $P b O M T$ genes in order to support the utility of the phylogenetic tree in the clarification of evolution relationships between $P b O M T s$. The results showed that these gene structures were very similar within the same subtypes, which revealed their close evolutionary relationship. Based on the number of introns in pear OMT genes, the OMTs can be divided into five types. Among 26 OMT genes, except eight introns that were located in PbCCOMT6, in the rest of of the family numbers, there were 4 genes with 4 introns, 11 genes with 3 introns, 6 genes with 2 introns, and 4 genes with 1 intron.

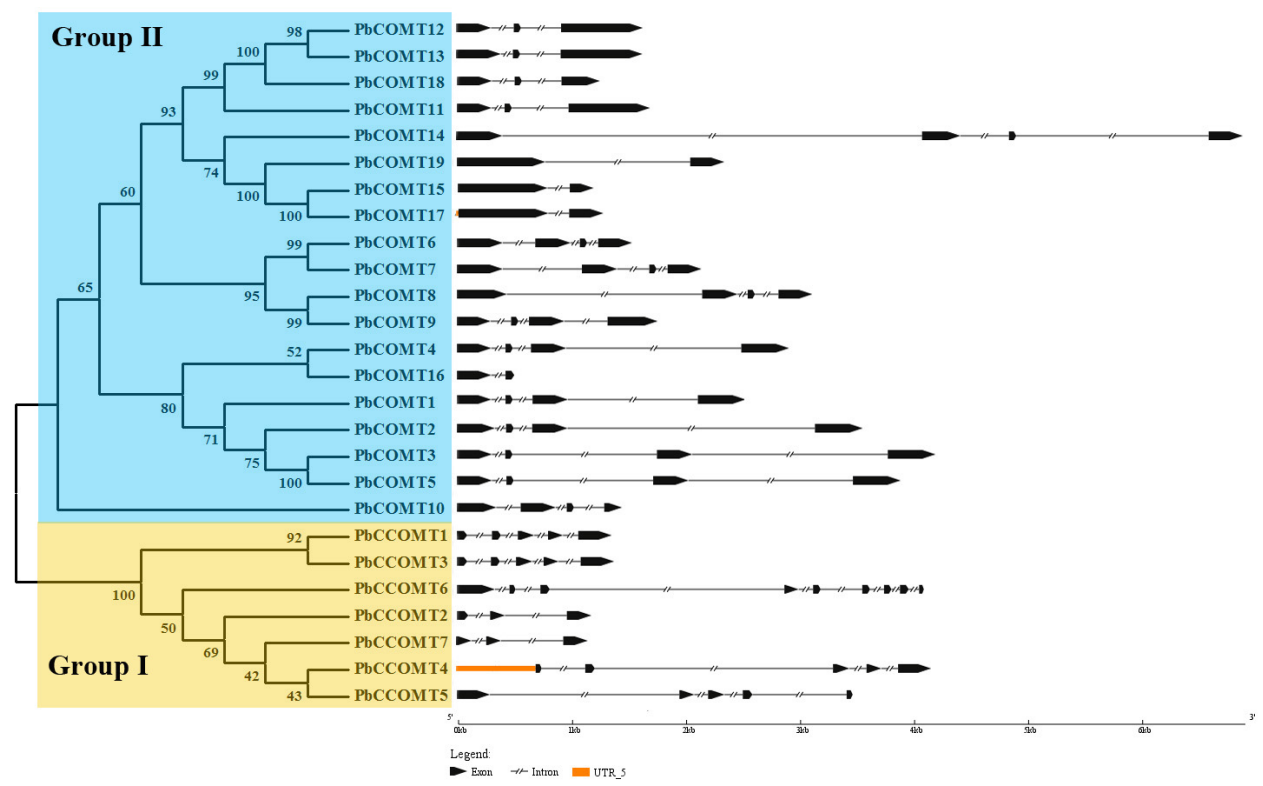

Figure 1. Phylogenetic relationships and gene structure of pear OMT proteins.

To further clarify the genetic relationships and to predict the biological function of OMT-gene products, $87 \mathrm{OMT}$ proteins from different species were used to construct a NJ tree 
(Figure 2). These proteins can be divided into two classes (classes I and II). Class I consists of all CCOMT genes, which are further divided into two subclasses (subclass I-a and I-b).

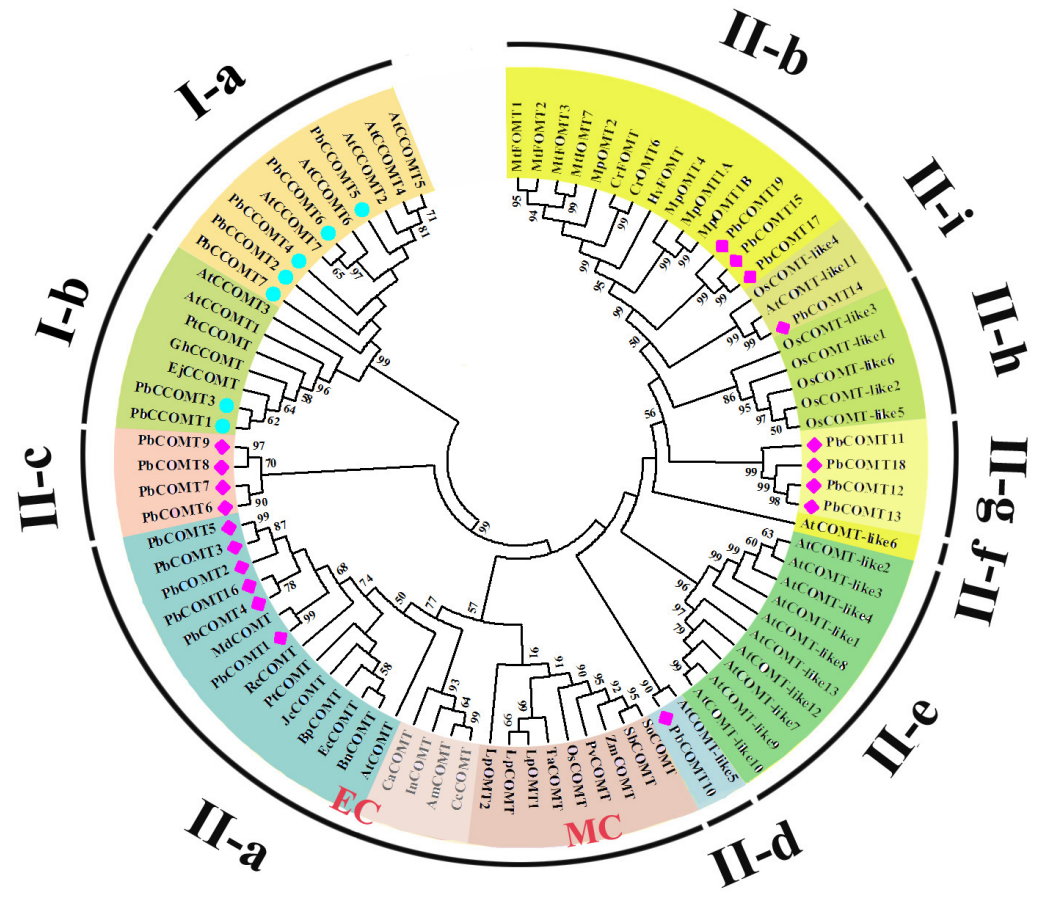

Figure 2. Neighbor-joining phylogenetic tree based on 87 OMT protein sequences from various plants. Diamonds represent PbCOMTs; blue dots represent PbCCOMTs. COMTs from subclass II-a were divided into two groups: a monocot cluster (MC) and a eudicot cluster (EC). Navy blue and pale pink shading represents the EC, pink shading represents the MC.

Five Arabidopsis CCOMTs and five pear CCOMTs were included in subclass I-a. Subclass I-b included two PbCCOMTs and two AtCCOMTs. Among them, AtCCOMT3 (At4g26220) is associated with the methylation of flavonone and flavanonol (Wils et al., 2013). AtCCOMT1 and PtCCOMT are associated with lignin synthesis (Do et al., 2007; Du et al., 2013), PbCCOMT1 and PbCCOMT3 gathered for a subclass with these two genes that may imply they are involved in lignin metabolism process.

Class II included all COMTs, which were further divided into nine subclasses (II-a-i). Pear COMTs were distributed in subclasses II-a, -b, -c, -d, -g, and -i. Within subclass II-c and -g, eight pear COMTs were included. Only AtCOMT- or OsCOMT-like genes were included in subclasses II-e, -f, and $-\mathrm{h}$, which is likely to be associated with species differentiation. PbCOMT15, -17, and -19 were included in subclass II-b, which belongs to the flavonoid OMTs, suggesting that these three PbCOMTs may be associated with flavonoid methylation (Kim et al., 2010; Sung et al., 2011). Most of the genes in subclass II-a are involved in lignin biosynthesis and include sequences from both monocots and eudicots, which could be further subdivided into two groups. The eudicot cluster could clearly be divided into two clades due to species variation. PbCCOMTs were grouped in subclass II-a of the eudicot cluster. Hirano et al. (2012) classified lignin metabolism related ZmCOMT, SbCOMT, OsCOMT, LpCOMT, 
LpCOMT1, LpCOMT3, AtOMT as true COMTs, including PbCOMT1 5 and PbCOMT16. There is preliminary evidence that one pear COMT (accession No. KC905086), PbCOMT1, is associated with lignin metabolism. The functions of the other five PbCOMTs in this group require further investigation.

The NJ tree also showed that PbCOMT14 and OsCOMT-like4 are closely related to AtCOMT-like11. Although some OMT genes in rice and pear could be grouped with AtOMTs, the bootstrap values in the tree nodes were low. For example, the bootstrap value of the PbCCOMT6 containing clade was only $65 \%$.

\section{Chromosomal localization and duplication of $O M T$ genes in pear}

To define the distribution of PbOMTs on chromosomes, and to analyze gene duplication events, a chromosome location analysis was performed. A total of 21 of $26 O M T$ genes were distributed on chromosomes 1, 5, 7, 9, 10, 12, 15, 16, and 17 (Figure 3), and most were located at the end of the chromosomes. As shown in Figure 3, chromosome 7 contains the maximum number of genes (four), with one each on chromosomes 5,12 , and 16 . The majority of these COMT genes are present as clusters in the pear genome. There is one gene cluster on chromosomes $1,7,9,10$, and 15, respectively. PbCCOMT4 forms a gene cluster with PbCOMT15 and PbCOMT17, suggesting their close relationship or co-regulation.

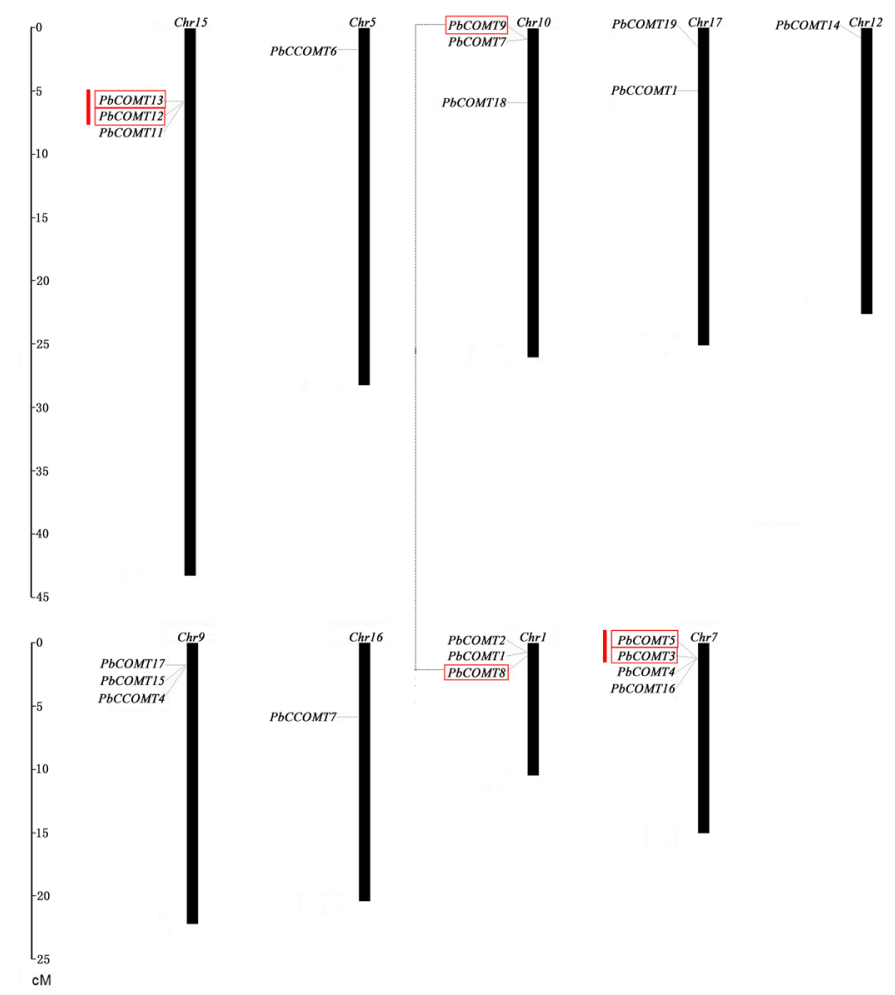

Figure 3. Chromosomal locations of pear $O M T$ genes on nine chromosomes. Genes involved in segmental duplication are joined by dashed lines, and the red rectangle indicates the gene cluster on each chromosome.

Genetics and Molecular Research 15 (3): gmr.15038664 
Based on the evolutionary relationships and chromosomal distribution of the PbOMT genes, one gene pair involved in segmental duplication and two pairs involved in tandem duplication were identified (Table 2). Generally, a Ka/Ks ratio of $>1$ indicates accelerated evolution with a positive selection, a ratio of 1 indicates pseudogenes with neutral selection, while a ratio $<1$ indicates functional constraints with negative or purifying gene selection. In this study, the $\mathrm{Ka} / \mathrm{Ks}$ ratios of three gene pairs were all less than 1 , indicating that their evolution was strongly influenced by purifying selection pressure although there was no strong differentiation for function.

Table 2. $\mathrm{Ka} / \mathrm{Ks}$ analysis for duplicated $O M T$ genes in pear.

\begin{tabular}{l|l|c|c|c|c|l}
\hline Duplicated gene 1 & Duplicated gene 2 & Ka & Ks & Ka/Ks & Purifying selection & Duplicated type \\
\hline PbCOMT8 & PbCOMT9 & 0.043 & 0.150 & 0.287 & Yes & Segmental \\
\hline PbCOMT3 & PbCOMT5 & 0.027 & 0.047 & 0.574 & Yes & Tandem \\
\hline PbCOMT12 & PbCOMT13 & 0.030 & 0.065 & 0.461 & Yes & Tandem \\
\hline
\end{tabular}

\section{Sequence conservation analysis}

To further unrevealing the relationship among the members of $O M T$ gene family, the distribution of the conserved motifs in individual member was analyzed using MEME software online. A total of 12 conservative motifs were identified (Figure 4 and Figure S1).

In addition, the OMT family could be divided into COMT and CCOMT types, based on clear differences in their conserved motifs. Using the Pfam software, these conserved motifs were further annotated. As shown in Figure 4 and Table 3, motifs 1-4 encode a methyltransf 2 domain, which only exists in PbCOMTs, and is mostly found in the middle of the sequence. All the PbCOMT proteins include these four motifs, except for PbCOMT16.

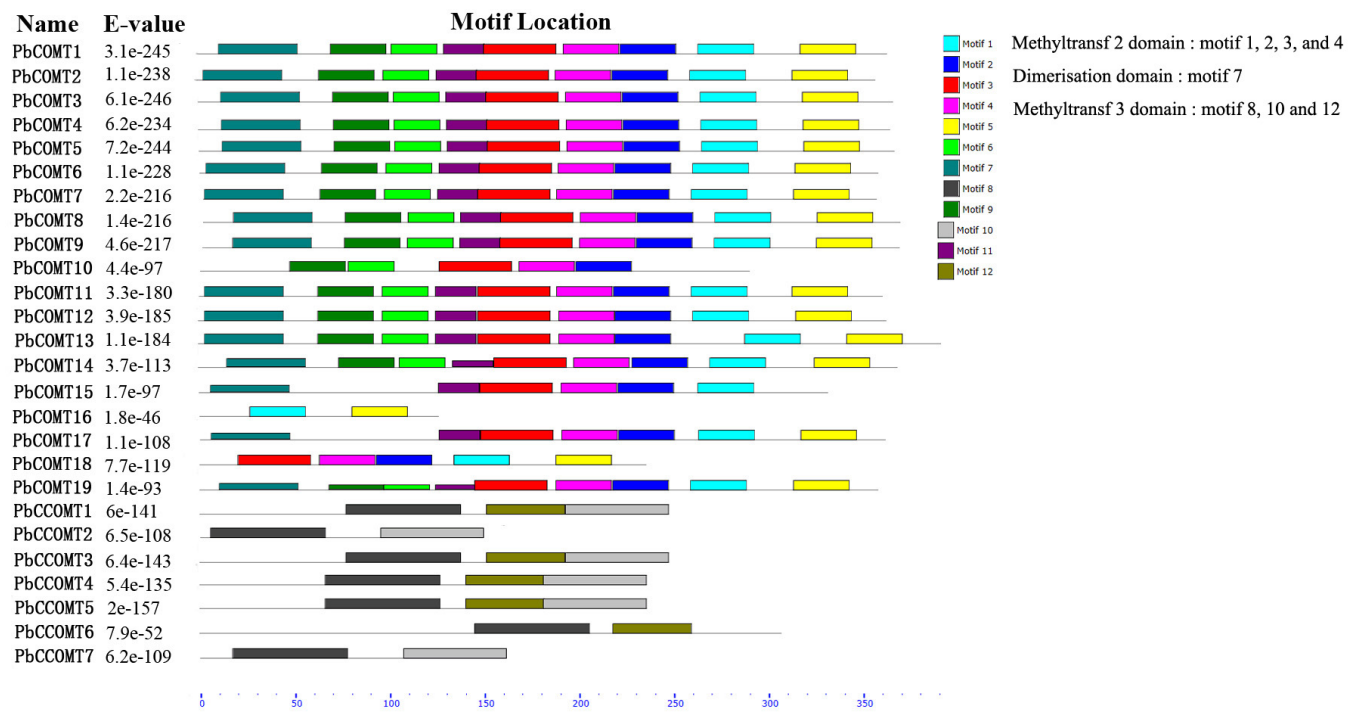

Figure 4. Distribution of 12 putative conserved motifs in pear OMT proteins.

Genetics and Molecular Research 15 (3): gmr.15038664 
Table 3. Motif composition of OMT proteins from pear.

\begin{tabular}{l|c|l}
\hline Motif No. & Width & Conserved amino acid sequences \\
\hline 1 & 29 & WICHDWSDDHCIKLLKNCWNAIPDNGKVI \\
\hline 2 & 29 & IKGINFDLPHVIKHAPQYPGVEHVGGDMF \\
\hline 3 & 38 & PFNKAHGMTTFEYHSTDPRFNQVFNKAMACHSTICMKK \\
\hline 4 & 29 & YKGFEGVTQLVDVGGGTGNAIWMIVSKYP \\
\hline 6 & 29 & MMAQNPGGKERTEEEFMALAKGAGFQGFR \\
\hline 7 & 24 & RLYGLTPVCKCFVTNEDGVSLAPL \\
\hline 9 & 41 & QRREEENYCFAMQLANSSVLQMVMQAAIELDVFDIMAKAGP \\
\hline 10 & 60 & MNAKKTMELGVYTGYSLLTTALALPDDGKITAIDVNKEAYEVGLPFIQKAGVEHKINFCQ \\
\hline 11 & 29 & DAPVMLDRMLRLLASYSILTCSLRTCPDD \\
\hline 12 & 54 & WFGTVAEPEENVQEYARYYRKHVLELNSFLAADPRIELCMVSVGDGLTLCRRLC \\
\hline
\end{tabular}

Motif 7 encodes a dimerization domain, which is also only present in PbCOMTs. The dimerization domain is annotated using Pfam as follows: it was found at the N-terminal of OMT in many plants, indicating its relevance to the formation of protein dimers. Accordingly, it is likely that COMT proteins are only present in monomeric forms because of the absence of a dimerization domain. Motifs 8, 10, and 12 encode a methyltransf 3 domain, which is only found in PbCCOMTs. Therefore, motifs 8,10 , and 12 could be used as feature motifs to distinguish COMTs and CCOMTs. Other motifs, such as 5, 6, 9, and 11 only exist in PbCOMTs, and their functions are unknown. Next, we used the OMT orthologs to construct the interaction network using the STRING software in Arabidopsis (Figure S2). OMT1 (PbCOMT1) was related to AT1G15950, 4CL2 (Pb4CL) and CCoAOMT1 (PbCCOMT1; PbCCOMT3).

Based on the analysis of conserved motifs, the sequence features of the two major types of genes in the OMT family, the COMTs and CCOMTs, were analyzed at the protein level by multiple comparison (Figure 5). The results of previous studies have found that the conserved domain in the COMT gene possesses the following motifs: $(\mathrm{V}, \mathrm{I}, \mathrm{L})(\mathrm{V}, \mathrm{L})(\mathrm{D}, \mathrm{K})$ (V, I) GGXX (G, A), GINFDLPHV, EHVGGDMF, NGKVI, and GGKERT (Joshi and Chiang, 1998). As shown in Figure 5, the five elements found in the PbCOMT proteins are relatively well conserved, with the exception of PbCOMT16, in which the first two motifs have been lost. Thus, these specific motifs could be used as features to identify COMT genes. Among the different $\mathrm{PbCOMT}$ proteins, the amino acid composition of these five motifs differed because of their specific substrates.

In plants, CCOMT has been found with three SAM-binding motifs, namely A, B, C, and other characteristic motifs, namely E, H, G, D, F (Joshi and Chiang, 1998). In the present study, these eight motifs were also identified in PbCCOMTs, with the loss of motif $\mathrm{D}$ in PbCCOMT7 and motif $\mathrm{G}$ in PbCCOMT2 and PbCCOMT7. It is likely that the differential distribution of motifs among the PbCCOMTs will be related to their functional differentiation. All screened PbOMTs possess the characteristic structure of full-length OMT proteins, which indicates that the results obtained by screening and identification are reliable.

\section{Identification and spatio-temporal expression analysis of candidate PbCCOMTs in $P$. bretschneideri cv. Dangshan Su}

To date, evidence has clearly shown that the COMT and CCOMT genes from Arabidopsis and maize are associated with lignin biosynthesis (Do et al., 2007; Li et al., 2013).

Genetics and Molecular Research 15 (3): gmr.15038664 

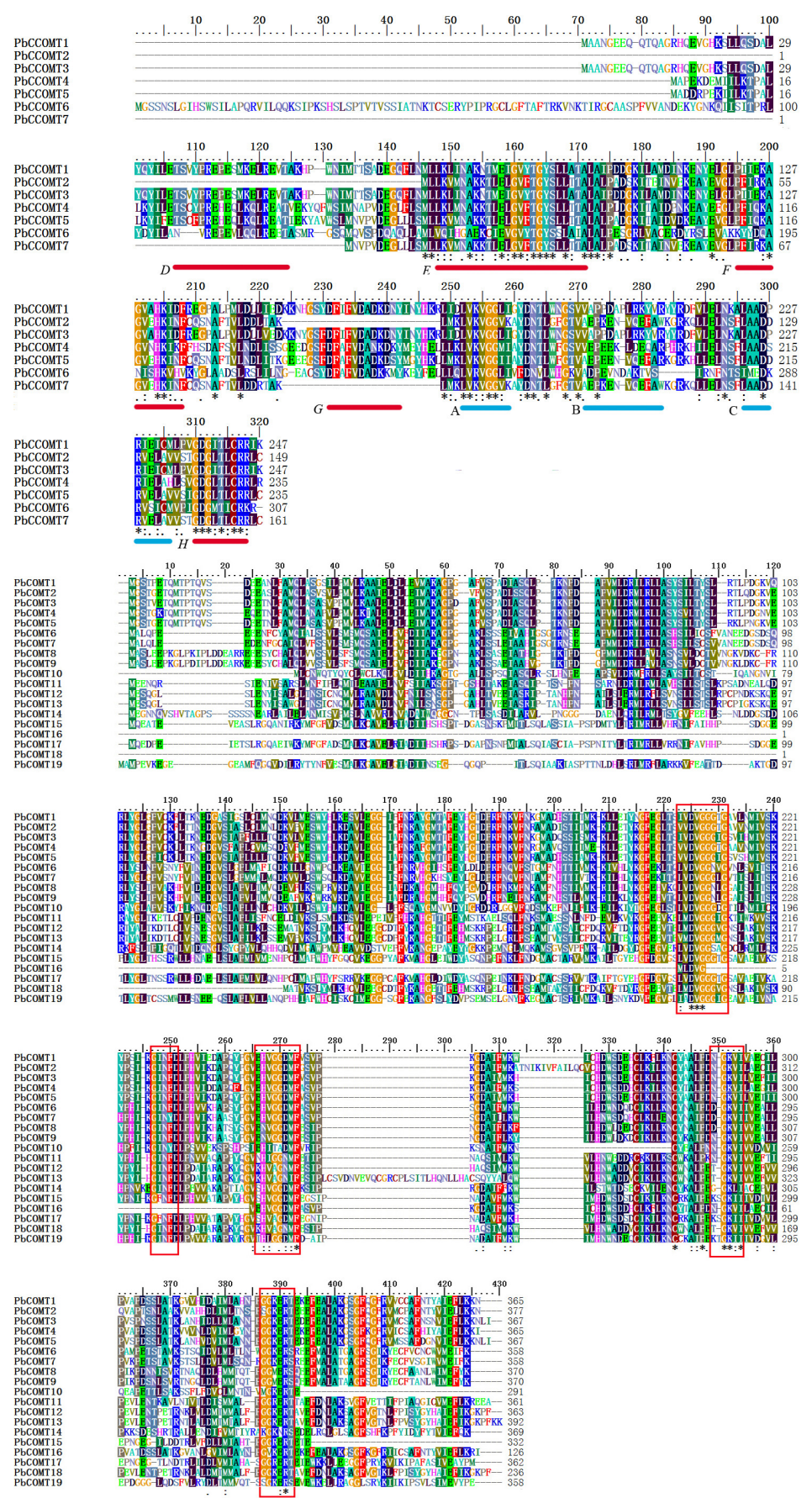

Figure 5. Multiple sequence alignment of $O M T$ proteins from pear, maize, and Arabidopsis. A: CCOMTs; B: COMTs. Conserved sequence motifs in COMT are boxed and bolded; three SAM-binding motifs, A, B, and C, are indicated below; CCOMT signature motifs are indicated as D, E, F, G, and H. 
In order to screen pear CCOMT genes related to lignin biosynthesis, Iden and Sim in Sequence Manipulation Suite methods were used to analyze the identity and similarity of seven pear CCOMT genes and to compare with those from Arabidopsis and maize (Table 4). Comparisons based on amino acid sequence further demonstrate that PbCCOMT1 and PbCCOMT3 are more similar to AtCCOMT1 and ZmCCOMT than to the other PbCCOMTs, indicating that PbCCOMT1 and PbCCOMT3 may act as CCOMT enzymes. This was consistent with the results of previous evolutionary tree clustering in this study. Therefore, PbCCOMT1 and $\mathrm{PbCCOMT3}$ were used as candidate genes for further study.

Table 4. Sequence identity and similarly among Arabidopsis, maize, and pear CCOMT protein homologs.

\begin{tabular}{l|c|c}
\hline Iden/Sim $(\% / \%)$ & AtCCOMT1 & ZmCCOMT \\
\hline PbCCOMT1 & $85 / 86$ & $37 / 81$ \\
\hline PbCCOMT2 & $31 / 33$ & $76 / 35$ \\
\hline PbCCOMT3 & $85 / 86$ & $51 / 56$ \\
\hline PbCCOMT4 & $49 / 54$ & $48 / 53$ \\
\hline PbCCOMT5 & $46 / 50$ & $29 / 35$ \\
\hline PbCCOMT6 & $27 / 34$ & $33 / 37$ \\
\hline PbCCOMT7 & $33 / 36$ & \\
\hline
\end{tabular}

Iden: identity; Sim: similarly.

The amino acid sequences of PbCCOMT1 and PbCCOMT3 were aligned with those of three other CCOMTs related to lignin biosynthesis, and using the ESPript tool, the distribution of secondary structure elements was screened (Figure 6). Higher conservation was observed among the five CCOMTs. The secondary structures of PbCCOMT1 and PbCCOMT3 are mainly composed of $\alpha$ helices and random curls, with some stretching sequences and $\beta$ angles. The secondary structure of the proteins encoded by PbCCOMT1 and PbCCOMT3 contain $36.44 / 34.01 \% \alpha$ helix, $21.05 / 23.89 \%$ extension chain, $12.96 / 11.34 \% \beta$ angle, and $29.55 / 30.77 \%$ random curl, respectively.

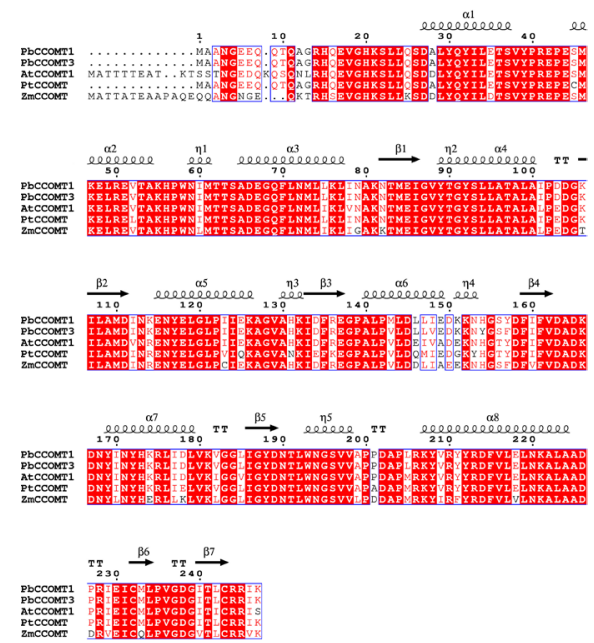

Figure 6. Alignment of the protein sequences encoded by $P b C C O M T 1$ and $P b C C O M T 3$ and other lignin CCOMTs. Secondary structure elements extracted from the protein structure of MsCCOMT (PDB id code: 1SUI) are indicated on top of the alignment using Espript. The arrows indicate $\beta$-sheet structure, the helices indicate helical structure motifs, and TT indicates turns.

Genetics and Molecular Research 15 (3): gmr.15038664 
The promoter sequences in $P b C C O M T 1$ and $P b C C O M T 3$ affects their expression. Both the PbCCOMT1 and PbCCOMT3 promoters possess light-responsive elements (Table 5), indicating their expression may be affected by light.

Table 5. Nucleotide motifs identified in promoter regions of putative lignin CCOMT genes in pear.

\begin{tabular}{|c|c|c|c|}
\hline Motif name & \multicolumn{2}{|c|}{$\begin{array}{c}\text { Motif location } \\
\text { PbCCOMTI PbCCOMT3 }\end{array}$} & Motif function \\
\hline AC-I & 1298 & 178,1290 & Most of the MYBs in this network can bind to the AC elements (I, II, and III) in the promoters \\
\hline AC-II & 1299,1300 & 1291 & of lignin biosynthesis genes and regulate their expression \\
\hline WUN-motif & - & 273 & Wound-responsive element \\
\hline TGACG-motif & 796 & 525,782 & Cis-acting regulatory element involved in MeJA-responsiveness \\
\hline MBS & - & 1146 & MYB Binding Site involved in drought-inducibility \\
\hline Sp1 & 547 & 134 & Light responsive element \\
\hline TC-rich repeats & 1444 & 405,1432 & Cis-acting element involved in defense and stress responsiveness \\
\hline GARE-motif & 440,1153 & 261 & Gibberellin-responsive element \\
\hline HSE & 462,521 & 965 & Cis-acting element involved in heat stress responsiveness \\
\hline LTR & - & 749 & Cis-acting element involved in low-temperature responsiveness \\
\hline TCA-element & $\begin{array}{c}45,154,237,810, \\
1143\end{array}$ & $\begin{array}{c}226,1203, \\
1204,1431,1471\end{array}$ & Cis-acting element involved in salicylic acid responsiveness \\
\hline
\end{tabular}

MBS: MYB binding site; HSE: heat shock element; LTR: low temperature responsive.

In addition, the two $P b C C O M T$ promoters also contain a large number of cis-elements for biological and abiotic stress responses, these include a WUN-motif, MBS, TC-rich repeats, HSE, LTR, TCA-element, and hormone-response related elements, such as those sensitive to methyl jasmonic acid (MeJA), salicylic acid, and gibberellin acid. Moreover, PbCCOMT1 and $P b C C O M T 3$ have AC components, which are generally associated with the regulation of lignin metabolism by combining MYB transcription factors (Raes et al., 2003; Liu et al., 2015). This indicates that the two genes play an important role in lignin biosynthesis.

Semi-quantitative RT-PCR was used to analyze the expression of candidate pear CCOMTs in different plant parts and at different development periods. As shown in Figure 7, expression of PbCCOMT1 and PbCCOMT3 was detected in the mature leaves, young stems, buds, and fruits. This may be because of the high lignin content in stems, therefore the expression of these genes are higher in stems than buds and mature leaves.

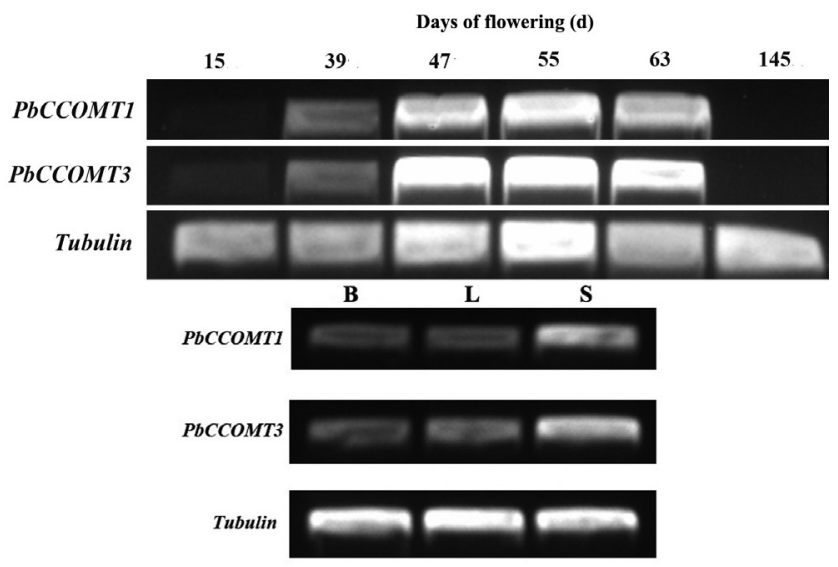

Figure 7. Expression patterns of candidate CCOMT genes in pear. PbCCOMT1 and PbCCOMT3 expression patterns in pulp during the development of pear fruits. PbCCOMT1 and PbCCOMT3 expression patterns in pear buds (B), mature leaves (L), and young stems (S). 
The expression of $P b C C O M T 1$ and $P b C C O M T 3$ followed similar trends in pear fruits. Low levels of these genes were present in fruit at 15 and $145 \mathrm{DAF}$. The expression levels in fruit from 47 to 63 DAF were significantly higher than those in mature leaves, young stems, and buds. PbCCOMT1 was maximally expressed $55 \mathrm{DAF}$. The levels of PbCCOMT3 expression between 47 and $63 \mathrm{DAF}$ were higher than those of $P b C C O M T 1$. These results are consistent with the trend observed in pear for lignin and stone cell contents (Cai et al., 2010; $\mathrm{Lu}$ et al., 2011), indicating that the two candidate CCOMT genes are probably involved in stone cell development and lignin biosynthesis in DangShan Su pear fruit.

\section{DISCUSSION}

Various members of the OMT gene family are involved in multiple branched pathways in phenylpropanoid metabolism and are mainly responsible for modification of plant secondary metabolite methylation. Two categories of OMTs exist: COMTs and CCOMTs. During lignin metabolism, COMT catalyzes 5-hydroxy-coniferyl aldehyde, caffeic acid, and 5-hydroxyconiferyl alcohol methylation to generate ferulic acid, sinapic alcohol, and sinapic aldehyde in the synthesis of S-lignin, respectively (Osakabe et al., 1999), CCOMT is responsible for transferring caffeoyl CoA to feruloyl CoA, and the latter subsequently participates in G-lignin synthesis.

The recent completion of whole genome sequencing in Arabidopsis (Raes et al., 2003), Populus trichocarpa (Shi et al., 2010), and Eucalyptus grandis (Carocha et al., 2015) has shown that these model organisms contain COMTs and CCOMTs. A total of 13 COMT-like genes and seven CCOMT genes exist in Arabidopsis. In the present study, AtCOMT-like5, -6, and -11 were grouped along one evolutionary branch, and the remaining 10 genes were grouped in another class, similar to previously reports (Raes et al., 2003). Among seven CCOMTs, only AtCCOMT1 belongs to the true CCOMT clade involved in lignin biosynthesis (Sibout et al., 2005; Do et al., 2007). PbCCOMT1 and PbCCOMT3 were grouped with AtCCOMT1, implying that they have a role in lignin metabolism. Although the functions of other members of this gene family remain unclear, AtCCOMT2, and AtCCOMT4-7, were expressed at different development periods in inflorescence stems (Raes et al., 2003). Six CCOMTs and 24 COMTS were isolated from P. trichocarpa and seven COMTs and two CCOMTs were isolated from $E$. grandis, respectively. In most plants, including pear, there are more members of COMTs than there are of CCOMTs. In this study, 19 PbCOMTs and seven PbCCOMTs were identified. CCOMTs should have different functions, such as involvement in lignin and flavonoid biosynthesis in pears. These results are consistent with previous reports that some CCOMTs can catalyze the synthesis of flavonoids in Arabidopsis and Camellia sinensis (Wils et al., 2013; Zhang et al., 2015).

Analysis of chromosomal localization indicated that members of the PbOMT gene family are distributed in the pear genome in the form of gene clusters. Together with the phylogenetic relationship, these results showed that gene duplication events within the $O M T$ gene family played a significant role in genome expansion in pear. In the present study, characteristics of PbOMTs were analyzed based on multiple comparisons of aminoacid sequences. The results from the sequence comparison and analysis of the conservative motifs, showed that both PbCOMTs and PbCCOMTs possessed the conserved domains shared by these proteins in other species. However, different members of the PbOMT gene family diverged and obtained various functions during the course of evolution. Generally, it is thought

Genetics and Molecular Research 15 (3): gmr.15038664 
that products of genes with similar sequences should carry similar functions. We found that PbCCOMT1 and PbCCOMT3 were highly similar to AtCCOMT1 and ZmCCOMT, and showed that PbCCOMT1 and PbCCOMT3 are involved in lignin metabolism in the same way as their homologous proteins AtCCOMT1 and ZmCCOMT.

The AC, MeJA-, and light-responsive elements in PbCCOMT1 and PbCCOMT3 were identified by cis-element analysis. Gharechahi et al. (2013) identified different CCOMT proteins from the hairy root of Silybum marianum L. treated by MeJA. Wang et al. (2013) found that the contents of stone cells and lignin decreased by 31.6 and $13.9 \%$, respectively, and that the quantity and diameter of stone cells was reduced by 28.0 and $45.8 \%$, respectively, in bagged pear fruits. This is likely because bagging affects the light intensity. Thus, MeJA and light might affect the expression of CCOMTs during stone cell development. During production, we can attempt to control light levels or spray MeJA onto the fruits to control the stone cell content. In addition, the regulatory function of AC elements upstream of genes related to lignin synthesis has been shown (Zhao and Dixon, 2011). AtCCOMT1 in A. thaliana possesses this $\mathrm{AC}$ element and has been found to be involved in lignin synthesis (Raes et al., 2003; Do et al., 2007). Thus, PbCCOMT1 and PbCCOMT3 both contain AC elements and may participate in lignin synthesis in pear.

Semi-quantitative RT-PCR showed that the expression of two CCOMT genes ( $P b C C O M T 1$ and $P b C C O M T 3$ ) was associated with stone cell and lignin contents during fruit development. These results suggest that PbCCOMT1 and PbCCOMT3 play key roles in the development of stone cells in Dangshan Su pear. High levels of PbCCOMT1 and PbCCOMT3 expression in stems is consistent with the expression of PtCCOMT in Populus tomentosa. High levels of CCOMT expression is associated with xylem development in woody plants (Shi et al., 2010; Yang et al., 2014). At present, many studies have used genetic engineering to suppress CCOMT expression to downregulate lignin (Wagner et al., 2011; Li et al., 2013; Pang et al., 2014; Zhang et al., 2014). In the present study, two CCOMT genes (PbCCOMT1 and $P b C C O M T 3$ ) involved in lignin biosynthesis have been identified. Subsequent research will focus on isolating the two genes and analyzing their function in lignin biosynthesis using transgenic methods. These genes could be used as targets for antisense and interference RNA technology to reduce the lignin content and production of stone cells and therefore increasing the quality of pear fruit.

\section{Conflicts of interest}

The authors declare no conflict of interest.

\section{ACKNOWLEDGMENTS}

Research supported by grants from the National Natural Science Foundation of China (\#31171944 and \#30771483).

\section{REFERENCES}

Bailey TL, Williams N, Misleh C and Li WW (2006). MEME: discovering and analyzing DNA and protein sequence motifs. Nucleic Acids Res. 34: W369-W373. http://dx.doi.org/10.1093/nar/gkl198

Bjellqvist B, Hughes GJ, Pasquali C, Paquet N, et al. (1993). The focusing positions of polypeptides in immobilized

Genetics and Molecular Research 15 (3): gmr.15038664 
$\mathrm{pH}$ gradients can be predicted from their amino acid sequences. Electrophoresis 14: 1023-1031. http://dx.doi. org/10.1002/elps.11501401163

Cai YP, Li GQ, Nie JQ, Lin Y, et al. (2010). Study of the structure and biosynthetic pathway of lignin in stone cells of pear. Sci. Hortic. 125: 374-379. http://dx.doi.org/10.1016/j.scienta.2010.04.029

Carocha V, Soler M, Hefer C, Cassan-Wang H, et al. (2015). Genome-wide analysis of the lignin toolbox of Eucalyptus grandis. New Phytol. 206: 1297-1313. http://dx.doi.org/10.1111/nph.13313

Choi JH and Lee SH (2013). Distribution of stone cell in Asian, Chinese, and European pear fruit and its morphological changes. J. Appl. Bot. Food Qual. 86: 185-189.

Do CT, Pollet B, Thévenin J, Sibout R, et al. (2007). Both caffeoyl Coenzyme A 3-O-methyltransferase 1 and caffeic acid O-methyltransferase 1 are involved in redundant functions for lignin, flavonoids and sinapoyl malate biosynthesis in Arabidopsis. Planta 226: 1117-1129. http://dx.doi.org/10.1007/s00425-007-0558-3

Du Q, Gong C, Pan W and Zhang D (2013). Development and application of microsatellites in candidate genes related to wood properties in the Chinese white poplar (Populus tomentosa Carr.). DNA Res. 20: 31-44. http://dx.doi. org/10.1093/dnares/dss031

Gharechahi J, Khalili M, Hasanloo T and Salekdeh GH (2013). An integrated proteomic approach to decipher the effect of methyl jasmonate elicitation on the proteome of Silybum marianum L. hairy roots. Plant Physiol. Biochem. 70: 115-122. http://dx.doi.org/10.1016/j.plaphy.2013.05.031

Goujon T, Sibout R, Pollet B, Maba B, et al. (2003). A new Arabidopsis thaliana mutant deficient in the expression of O-methyltransferase impacts lignins and sinapoyl esters. Plant Mol. Biol. 51: 973-989. http://dx.doi. org/10.1023/A:1023022825098

Hirano K, Aya K, Kondo M, Okuno A, et al. (2012). OsCAD2 is the major CAD gene responsible for monolignol biosynthesis in rice culm. Plant Cell Rep. 31: 91-101. http://dx.doi.org/10.1007/s00299-011-1142-7

Kim BG, Sung SH, Chong Y, Lim Y, et al. (2010). Plant flavonoid O-methyltransferases: substrate specificity and application. J. Plant Biol. 53: 321-329. http://dx.doi.org/10.1007/s12374-010-9126-7

Kong X, Lv W, Jiang S, Zhang D, et al. (2013). Genome-wide identification and expression analysis of calcium-dependent protein kinase in maize. BMC Genomics 14: 433. http://dx.doi.org/10.1186/1471-2164-14-433

Li X, Chen W, Zhao Y, Xiang Y, et al. (2013). Downregulation of caffeoyl-CoA O-methyltransferase (CCoAOMT) by RNA interference leads to reduced lignin production in maize straw. Genet. Mol. Biol. 36: 540-546. http://dx.doi. org/10.1590/S1415-47572013005000039

Liu JY, Osbourn A and Ma PD (2015). MYB transcription factors as regulators of phenylpropanoid metabolism in plants. Mol. Plant 8: 689-708. http://dx.doi.org/10.1016/j.molp.2015.03.012

Lu XP, Liu YZ, An JC, Hu HJ, et al. (2011). Isolation of a cinnamoyl CoA reductase gene involved in formation of stone cells in pear (Pyrus pyrifolia). Acta Physiol. Plant. 33: 585-591. http://dx.doi.org/10.1007/s11738-010-0583-x

Magali L, Patrice D, Gert T, Kathleen M, et al. (2002). PlantCARE, a database of plant cis-acting regulatory elements and a portal to tools for in silico analysis of promoter sequences. Nucleic Acids Res. 30: 325-327. http://dx.doi. org/10.1093/nar/30.1.325

Jin Q, Yan CC, Qiu JX, Zhang N, et al. (2013). Structural characterization and deposition of stone cell lignin in Dangshan Su pear. Sci. Hortic. 155: 123-130. http://dx.doi.org/10.1016/j.scienta.2013.03.020

Joshi CP and Chiang VL (1998). Conserved sequence motifs in plant S-adenosyl-L-methionine-dependent methyltransferases. Plant Mol. Biol. 37: 663-674. http://dx.doi.org/10.1023/A:1006035210889

Osakabe K, Tsao CC, Li L, Popko JL, et al. (1999). Coniferyl aldehyde 5-hydroxylation and methylation direct syringyl lignin biosynthesis in angiosperms. Proc. Natl. Acad. Sci. U. S. A. 96: 8955-8960. http://dx.doi.org/10.1073/ pnas.96.16.8955

Pang SL, Ong SS, Lee HH, Zamri Z, et al. (2014). Isolation and characterization of CCOAOMT in interspecific hybrid of Acacia auriculiformis x Acacia mangium - a key gene in lignin biosynthesis. Genet. Mol. Res. 13: 7217-7238. http:// dx.doi.org/10.4238/2014.September.5.7

Raes J, Rohde A, Christensen JH, Van de Peer Y, et al. (2003). Genome-wide characterization of the lignification toolbox in Arabidopsis. Plant Physiol. 133: 1051-1071. http://dx.doi.org/10.1104/pp.103.026484

Robert X and Gouet P (2014). Deciphering key features in protein structures with the new ENDscript server. Nucleic Acids Res. 42: 320-324. http://dx.doi.org/10.1093/nar/gku316

Rozas J and Rozas R (1995). DnaSP, DNA sequence polymorphism: an interactive program for estimating Population Genetics parameters from DNA sequence data. Comput. Applic. Biosci. 11: 621-625.

Shi R, Sun YH, Li QZ, Heber S, et al. (2010). Towards a systems approach for lignin biosynthesis in Populus trichocarpa: transcript abundance and specificity of the monolignol biosynthetic genes. Plant Cell Physiol. 51: 144-163. http:// dx.doi.org/10.1093/pcp/pcp175

Genetics and Molecular Research 15 (3): gmr.15038664 
Sibout R, Eudes A, Mouille G, Pollet B, et al. (2005). Cinnamyl alcohol dehydrogenase-C and -D are the primary genes involved in lignin biosynthesis in the floral stem of Arabidopsis. Plant Cell 17: 2059-2076. http://dx.doi.org/10.1105/ tpc. 105.030767

Stothard P (2000). The sequence manipulation suite: JavaScript programs for analyzing and formatting protein and DNA sequences. Biotechniques 28: 1102-1104.

Sung SH, Kim BG, Chong Y and Ahn JH (2011). Characterization of phenylpropanoid O-methyltransferase from rice: molecular basis for the different reactivity toward different Substrates. J. Plant Biol. 54: 314-320. http://dx.doi. org/10.1007/s12374-011-9169-4

Tamura K, Peterson D, Peterson N, Stecher G, et al. (2011). MEGA5: molecular evolutionary genetics analysis using maximum likelihood, evolutionary distance, and maximum parsimony methods. Mol. Biol. Evol. 28: 2731-2739. http://dx.doi.org/10.1093/molbev/msr121

van Parijs FRD, Ruttink T, Boerjan W, Haesaert G, et al. (2015). Clade classification of monolignol biosynthesis gene family members reveals target genes to decrease lignin in Lolium perenne. Plant Biol. 17: 877-892. http://dx.doi. org/10.1111/plb.12316

Wagner A, Tobimatsu Y, Phillips L, Flint H, et al. (2011). CCoAOMT suppression modifies lignin composition in Pinus radiata. Plant J. 67: 119-129. http://dx.doi.org/10.1111/j.1365-313X.2011.04580.x

Wang B, Zhang N, Yan CC, Jin Q, et al. (2013). Bagging for the development of stone cell and metabolism of lignin in Pyrus bretschneideri Dangshan Suli. Acta Hortic. Sin. 40: 531-539.

Wei H, Li W, Sun X, Zhu S, et al. (2013). Systematic analysis and comparison of nucleotide-binding site disease resistance genes in a diploid cotton Gossypium raimondii. PLoS One 8: e68435. http://dx.doi.org/10.1371/journal.pone.0068435

Wils CR, Brandt W, Manke K and Vogt T (2013). A single amino acid determines position specificity of an Arabidopsis thaliana CCoAOMT-like $O$-methyltransferase. FEBS Lett. 587: 683-689. http://dx.doi.org/10.1016/j. febslet.2013.01.040

Wu J, Wang ZW, Shi ZB, Zhang S, et al. (2012). The genome of the pear (Pyrus bretschneideri Rehd.). Genome Res. 23: 396-408. http://dx.doi.org/10.1101/gr.144311.112

Yan CC, Yin M, Zhang N, Jin Q, et al. (2014). Stone cell distribution and lignin structure in various pear varieties. Sci. Hortic. 174: 142-150. http://dx.doi.org/10.1016/j.scienta.2014.05.018

Yang XH, Li XG, Li BL and Zhang DQ (2014). Genome-wide transcriptional profiling reveals molecular signatures of secondary xylem differentiation in Populus tomentosa. Genet. Mol. Res. 13: 9489-9504. http://dx.doi. org/10.4238/2014.November.11.14

Zhang G, Zhang YJ, Xu JT, Niu XP, et al. (2014). The CCoAOMT1 gene from jute (Corchorus capsularis L.) is involved in lignin biosynthesis in Arabidopsis thaliana. Gene 546: 398-402. http://dx.doi.org/10.1016/j.gene.2014.05.011

Zhang Y, Lv HP, Ma CY, Guo L, et al. (2015). Cloning of a caffeoyl-Coenzyme A O-methyltransferase from Camellia sinensis and analysis of its catalytic activity. J. Zhejiang Univ. Sci. B. 16: 103-112. http://dx.doi.org/10.1631/jzus. $\underline{B 1400193}$

Zhao Q and Dixon RA (2011). Transcriptional networks for lignin biosynthesis: more complex than we thought? Trends Plant Sci. 16: 227-233. http://dx.doi.org/10.1016/j.tplants.2010.12.005

Zhao SG, Zhang JG, Zhao YP, Zhang YX (2013). New discoveries of stone cell differentiation in fruitlets of 'Yali' pears (Pyrus bretschneideri Rehd.). J. Food Agric. Enviro. 11: 937-942.

\section{Supplementary material}

Table S1. Sequences used for phylogenetic analyses of $O M T$ proteins.

Table S2. Primers used for semi-quantitative RT-PCR of PbCCOMT homologs. Tubulin (accession No. AB239680.1) served as the internal standard.

Figure S1. Detailed amino acid composition of the 12 motifs in PbOMT proteins.

Figure S2. Functional interaction networks of OMTs in pear according to orthologs in Arabidopsis.

Genetics and Molecular Research 15 (3): gmr.15038664 